\title{
SUR LA THÉORIE OSMOTIQUE DES PILES;
}

Par M. M. COUETTE.

Introduction. - La théorie osmotique des forces électromotrices créée par M. Walther Nernst( $\left.{ }^{1}\right)$, en 1889, est devenue, ce me semble, aujourd'hui, presque classique en Allemagne $\left({ }^{2}\right)$ et s'impose à l'attention en France $\left(^{3}\right)$. Or elle conduit à des conclusions opposées à celles des recherches expérimentales de nos compatriotes, relativement aux différences de potentiel au contact. La première colonne du tableau suivant donne, pour les différents contacts d'une pile de Daniell, les valeurs que M. Pellat $\left({ }^{4}\right)$ a déduites de la combinaison de ses propres expériences avec celles de M. Gouré de la Villemontée; la deuxième colonne donne les valeurs adoptées en Allcmagne $\left({ }^{5}\right)$.

$$
\begin{aligned}
& \mathrm{Zn} \mid \mathrm{SO}^{\mathrm{i}} \mathrm{Zn} \ldots \ldots .600 \quad 0 \quad 0^{\mathrm{v}, 524} \\
& \mathrm{SO}^{4} \mathrm{Zn} \mid \mathrm{SO}^{4} \mathrm{Cu} \ldots \ldots \ldots 0^{\mathrm{r}, 04 \mathrm{5} 4} \text { presque } 0
\end{aligned}
$$

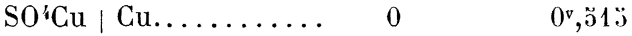

$$
\begin{aligned}
& \mathrm{Cu} \mid \mathrm{Zn} \ldots \ldots \ldots \ldots 0^{\mathrm{v}}, 664 \quad \text { presque } 0 .
\end{aligned}
$$

La considération de la pression osmotique, au lieu de la tension de vapeur, simplifie la démonstration de la relation établie par von Helmholtz $\left(^{\boldsymbol{\theta}}\right)$ entre la force électromotrice d'une pile et la concentration des solutions qui en font partie. Mais l'application rigoureuse des principes de la thermodynamique ne donne que la force électromotrice totale et ne suffit pas pour déterminer séparément les différences de potentiel aux divers contacts. M. Pellat a demandé cette dernière détermination à l'expérience; M. Nernst l'a déduite théoriquement de certaines considérations que nous exposerons plus loin.

(1) Die elektromotorische Wirksamkeit der Jonen (Zeitschrift für physikalische Chemie, t. IV, p. 129).

(2) $\mathrm{D}^{\mathrm{r}}$ F. HABer, Grundriss der technischen Elektrochemie, Leipzig, 1898 ; W. Ostwald, Grundriss der allgemeinen Chemie, $3^{\text {te }}$ auflage. Leipzig, 1899; etc. (On peut juger de l'importance attachée en Allemagne à cette théorie et du nombre des recherches qu'elle a inspirées par la lecture des analyses données ici même par M. Gunchaxt, des travaux que publie la Zeitschrift für physikalische Chemie. Voir, en particulier, le présent volume, p. 231 et suivantes. R.

(3) A. Holland, Revue générale des Sciences, t. 1X, p. 358 et p. $411 ;-i d .$, t. X, p. 939; 1898-1899; - M. LE Blanc, Rev. gén. des Sc., t. X, p. $725 ; 1899$.

(4) Bull. de la Soc. de Phys., 1890, p. 188.

(5) Di HaBer, loc. cit., p. 107.

(6) Berl. Sitzungsber., 1882, p. 836;-J. de Phys., 2e série., t. III, p. $396 ; 1884$. - Cours de Thermodynamique de M. Lippmann, p. 212. 


\section{II}

Théonie thermodinamique. - Lemme. - Quand la tempéralure $\mathrm{T}$ figure parmi les variables indépendantes, on peut écrire, pour chacune des expressions dF et $d \mathrm{Q}$, les conditions d'inte'grabilité relatives aux variables autres que $\mathrm{T}$.

Soient en effet :

et par suite :

$$
\begin{aligned}
& d \widetilde{C}=\mathbf{A} d x+\mathbf{B} d y+\ldots \mathrm{C} d \mathrm{~T} \\
& d \mathbf{Q}=\mathbf{L} d x+\mathbf{M} d y+\ldots \mathrm{N} d \mathrm{~T}
\end{aligned}
$$

$$
\begin{aligned}
& d \mathbf{U}=d \mathbf{T}+\mathbf{J} d \mathbf{Q}=(\mathbf{A}+\mathbf{J L}) d x+(\mathbf{B}+\mathbf{J M}) d y+\ldots(\mathbf{C}+\mathbf{J N}) d \mathbf{T} \\
& d \mathbf{S}=\frac{d \mathbf{Q}}{\mathrm{T}}=\frac{\mathrm{L}}{\mathrm{T}} d x+\frac{\mathbf{M}}{\mathbf{T}} d y+\ldots \frac{\mathbf{N}}{\mathrm{T}} d \mathrm{~T} .
\end{aligned}
$$

Les conditions d'intégrabilité de $d \mathbf{U}$ et de $d \mathbf{S}$ sont :

$$
\begin{aligned}
\frac{\partial \mathrm{A}}{\partial y}+\mathrm{J} \frac{\partial \mathrm{L}}{\partial y} & =\frac{\partial \mathrm{B}}{\partial x}+\mathrm{J} \frac{\partial \mathrm{M}}{\partial x}, \\
\frac{1}{\mathrm{~T}} \frac{\partial \mathrm{L}}{\partial y} & =\frac{1}{\mathrm{~T}} \frac{\partial \mathrm{M}}{\partial x},
\end{aligned}
$$

relativement aux variables $x$ et $y$; en les combinant, on trouve :

$$
\frac{\partial \mathrm{A}}{\partial y}=\frac{\partial \mathrm{B}}{\partial x}, \quad \frac{\partial \mathrm{M}}{\partial y}=\frac{\partial \mathrm{M}}{\partial x},
$$

et on obtient deux équations semblables pour tout couple de variables autres que $\mathbf{T}$.

Formule fondamentale. - Considérons d'abord une pile à un seul liquide et à dépolarisant solide. Donnons-lui pour pôles deux conducteurs creux, A et $\mathrm{B}$, formés d'un même métal quelconque. Nous transporterons, comme il nous plaira, de l'électricité de l'extérieur de l'un à l'intérieur de l'autre, au moyen d'un porteur de capacité infiniment pelite, de même mélal que les pôles, et fixé sur un manche isolant. Son mourement sera infiniment lent, afin que l'effet Joule soit négligeable, et les conditions de réversibilité, satisfaites.

Alors la différence de potentiel entre les pôles sera égale à la force électromotrice de la pile :

$$
\mathrm{V}_{\mathrm{B}}-\mathrm{V}_{\mathrm{A}}=\mathrm{E} \text {; }
$$

et le travail de la force appliquée par l'opérateur au porteur, pendant le transport de la quantité d'électricité $d m$ du pôle $\mathrm{B}$ au pôle $\mathrm{A}$, sera :

$$
\left(\mathrm{V}_{\mathrm{A}}-\mathrm{V}_{\mathrm{B}}\right) d m=-\mathrm{E}_{l} l m .
$$


D`autre part, le liquide de la pile communiquera, par l'intermédiaire d'une cloison semi-perméable, avec de l'eau pure contenue dans un corps de pompe. En déplaçant le piston, nous ferons passer, à volonté, de l'eau pure, dans un sens ou dans l'autre, à travers la cloison, et nous modificrons ainsi le volume $v$, et, par suite, la concentration de la solution électrolytique. La condition d'équilibre osmotique entre cette solution et l'eau de la pompe est que la pression de la première ait sur celle de la seconde un excès égal à la pression osmolique $\varpi$. Pour remplir la condition de réversibilité, nous donnerons au piston un mouvement assez lent pour que l'équilibre osmotique soit toujours infiniment près d'ètre réalisś. Alors, pendant l'accroissement $d v$, de volume de la solution, la somme des travaux de la pression atmosphérique $\mathrm{P}$, qui s'exerce à sa surface libre, et de la pression $\mathrm{P}-\overline{-}$, exercée par le piston sur l'eau de la pompe, sera :

$$
-\mathrm{P} d v+(\mathrm{P}--\varpi) d v=-\varpi d v .
$$

Prenons pour variables indépendantes la température $\mathbf{T}$ du système, le volume $v$ de la solution et la quantité $m$ d'électricité qui, depuis un certain instant initial, a traversé la pile en allant du pôle A au pôle B. Pour la transformation $d v$, $d m$, le travail des forces extérieures est :

$$
d \tau=-\mathbf{E} d m-\sigma d v ;
$$

et le lemme nous fournit la relation :

$$
\frac{\partial E}{\partial v}=\frac{\partial \sigma}{\partial m} .
$$

Nous désignerons par $\mathrm{N}$ le nombre total des molécules-grammes de sel contenues dans la dissolution, et par $\gamma$ sa concentration moléculaire, c'est-à-dire le nombre de molécules-grammes de sel qu'elle contient par centimètre cube, abstraction faite de toute dissociation. Evidemment :

$$
\mathbf{N}=v \gamma \cdot
$$

E ne dépend de $v$ que par l'intermédiaire de $\gamma$. On peut donc écrire :

$$
\frac{\partial \mathrm{E}}{\partial v}=\frac{\partial \mathrm{E}}{\partial \gamma} \cdot \frac{\partial \gamma}{\partial v}=\frac{\partial \mathrm{E}}{\partial \gamma}\left(-\frac{\gamma}{v}\right)
$$

car, lorsqu'on modifie $\gamma$ en changeant $v$, mais non $\mathrm{N}$, la différentia tion de l'équation (2) donne :

$$
0=\gamma d v+v d \gamma
$$


De même, $\sigma$ ne dépend de $m$ que par l'intermédiaire de $\gamma$. Le passage de la quantité d'électricité $d m$ à travers l'électrolyte augmen te de $d \mathrm{~N}$ le nombre de ses molécules, sans changer son volume, et la différentiation de l'équation (2) donne alors :

Par conséquent:

$$
\frac{\partial \mathrm{N}}{\partial m}=v \frac{\partial \gamma}{\partial m}
$$

$$
\frac{\partial \pi}{\partial m}=\frac{\partial \sigma}{\partial \gamma} \cdot \frac{\partial \gamma}{\partial m}=\frac{\partial \sigma}{\partial \gamma} \frac{1}{v} \cdot \frac{\partial N}{\partial m} .
$$

En substituant dans (1) les expressions (2) et (4), on obtient enfin :

$$
\frac{\partial \mathrm{E}}{\partial \gamma}=-\frac{1}{\gamma} \cdot \frac{\partial \pi}{\partial \gamma} \cdot \frac{\partial N}{\partial m},
$$

relation équivalente à celle de von Helmholtz .

Le facteur $\frac{\partial N}{\partial m}$ ne dépend pas de $\gamma$; et une simple quadrature donne :

$$
\mathrm{E}=-\frac{\partial \mathbf{N}}{\partial m} \int \frac{1}{\gamma} \cdot \frac{\partial \pi}{\partial \gamma} \cdot d \gamma
$$

Généralisation. - La même théorie s'applique sans difficulté aux piles à deux liquides, à plusieurs piles et généralement à plusieurs solutions électrolytiques, placées en série dans un même circuit. Il suffit d'adjoindre à chaque solution une cloison semi-perméable et une pompe; alors les volumes $v_{1}, v_{2}, v_{3}, \ldots$, des diverses solutions sont des variables in tépendantes; l'expression du travail devient :

$$
d E=-\mathrm{E} d m-\varpi_{1} d v_{1}-\varpi_{2} d v_{2} \ldots
$$

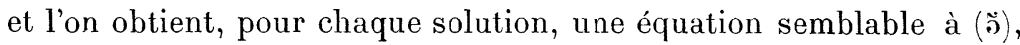
où $\mathrm{E}$ désigne toujours la force électronotrice totale de la série, au lieu que $\sigma, \gamma, \frac{\partial N}{\partial m}$ sont spéciaux à chaque solution. Connaissant ainsi les dérivées partielles $\frac{\partial E}{\partial \gamma_{1}}, \frac{\partial E}{\partial \gamma_{2}}$, etc., on arrive, par intégration, à la formule :

$$
\mathbf{E}=\Sigma\left[-\frac{\partial N}{\partial m} \int \frac{1}{\gamma} \frac{\partial \pi}{\partial \gamma} \cdot d \gamma\right] \text {. }
$$

Expression développée de $\frac{\partial \omega}{\partial \gamma}$ et calcul de lintégrale $\int \frac{1}{\gamma} \frac{\partial \omega}{\partial \gamma} d \%$ - 
D'après M. Arrhenius (1) les molécules des sels, acides ou hydrates en dissolution, sont plus ou moins complètement dissociées en ions, et chaque ion joue, au point de vue de la pression osmotique, le même rôle qu'une molécule non dissociée. On appelle degrí de dissociation ó le rapport du nombre de molécules dissociées au nombre total de molécules dissociées ou non; si donc une solution contient en tout $\mathrm{N}$ molécules, $\mathrm{N} \delta$ sont dissociées en ions, et $\mathrm{N}(1-\delta)$ ne le sont pas. Soit $j$ le nombre d'ions fournis par 1 molécule $\left(j=2\right.$ pour $\mathrm{NaCl}$ et pour $\mathrm{SO}^{4} \mathrm{Zn} ; j=3$ pour $\mathrm{ZnCl}^{2}$ et pour $\left.\mathrm{SO}^{4} \mathrm{H}^{2}\right)$; et soit 2 le nombre total d'ions et de molécules non dissociées qui se trouvent dans une dissolution, où le nombre des molécules primitives est $\mathrm{N}$. On trouve facilement :

$$
\jmath \tau=\mathrm{N} \delta j+\mathrm{N}(1-\delta)=\mathrm{N}[1+(j-1) \delta] .
$$

Quand la dissociation est complète (cas limite dont se rapprochent les solutions très diluées), $\delta=1$ et $\mathfrak{r}=\mathrm{N} j$.

D'autre part, d'après M. Van't Hoff $\left({ }^{2}\right)$, la pression osmotique $\varpi$ satisfait à une équation semblable à celle des gaz parfaits :

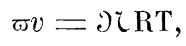

où $\mathscr{r}$ a la même signification que ci-dessus, et $\mathrm{R}$ représente la même constante que pour les gaz, soit 8,316 C. G. S.

Premier cas. - Dissociation complète : $\mathfrak{x}=\mathrm{N} j$.

et

$$
\varpi v=\mathbf{N} \mathbf{R} \mathbf{T}
$$

$$
\varpi=j \gamma \mathrm{RT}, \quad \text { puisque } \quad \frac{\mathrm{N}}{v}=\gamma \text {. }
$$

Alors :

et

$$
\frac{\partial \varpi}{\partial \gamma}=\mathrm{RT} j
$$

$$
\int \frac{1}{\gamma} \cdot \frac{\partial \omega}{\partial \gamma} d \gamma=j \mathrm{RT} \cdot \log \gamma+\mathrm{C} .
$$

Je désigne par log les logarithmes nepériens.

Deuxième cas. - Dissociation parlielle.

d'où :

$$
x=x[1+(j-1) \delta] .
$$

$$
\varpi=[1+(j-1) \delta] \curlyvee \mathrm{RT}^{\mathrm{T}} \text {; }
$$

(1) Zeitsch.f.ph. Ch., t. I, p. $631 ; 1881$.

(2) Zeitsch.f.ph. Ch., t. 1, p. 481; 1887. 
Le degré de dissociation $\delta$ et la concentration moléculaire $\gamma$ sont liés l'un à l'autre par la loi des masses (1). Soient $m_{1}$ et $m_{2}$ les masses des produits mis en liberté par une dissociation, $m_{3}$ la masse du reste non dissocié, le tout dans le volume $v$ à la température $\mathrm{T}$; cette loi s'exprime par l'égalité :

$$
\frac{m_{1} m_{2}}{m_{3}}=\mathrm{K} v
$$

où le coefficient $\mathrm{K}$, appelé constanle de dissociation, est fonction de $\mathrm{T}$, mais non de $v$. Dans le cas de la dissociation électrolytique, Nó molécules dissociées fournissent $\mathrm{Nò} \mathrm{groupes} \mathrm{cationiques} \mathrm{de} \mathrm{masse} \mu_{1}$ et $\mathrm{N} \delta$ groupes anioniques de masse $\mu_{2} ; \mathrm{N}(1-\delta)$ molécules de masse $\left(\mu_{1}+\mu_{2}\right)$ ne sont pas dissociées. On a donc :

$$
m_{1}=\mathrm{N} \delta \mu_{1}, \quad m_{2}=\mathrm{N} \delta \mu_{2}, \quad m_{3}=\mathrm{N}(1-\delta)\left(\mu_{1}+\mu_{2}\right) .
$$

En substituant ces valeurs dans (11), se rappelant que $\frac{N}{v}=\gamma$, et posant $\mathrm{K} \frac{\mu_{1}+\mu_{2}}{\mu_{1} \mu_{2}}=k$, on trouve :

$$
\gamma \delta^{2}=k(1-\delta):
$$

L'élimination de $\delta$ entre $(10)$ et $(12)$ donne :

Donc :

$$
\varpi=\mathrm{RT} \gamma-\frac{k(j-1)}{2} \cdot \mathrm{RT}+\frac{j-1}{2} \sqrt{k^{2}+4 k \gamma} \cdot \mathrm{RT} .
$$

et

$$
\frac{\partial \sigma}{\partial \gamma}=\mathrm{RT}+\frac{(j-1) k \mathrm{RT}}{\sqrt{k^{2}+4 k \gamma}},
$$

$$
\int \frac{1}{\gamma} \cdot \frac{\partial \sigma}{\partial \gamma} d \gamma=\mathbf{R T}\left[\log \gamma+(j-1) \log \frac{\sqrt{k^{2}+4 k \gamma}-k}{\sqrt{k^{2}+4 k \gamma}+k}\right]+\mathrm{C} .
$$

Expression díveloppée de $\frac{\partial \mathrm{N}}{\partial m}$. Elle dépend non seulement de la nature chimique de la solution considérée, mais encore de celles des électrodes ou des autres solutions en contact avec elle. Nous la formerons pour les cas les plus importants.

$\mathbf{1}^{0}$ Pile à dépolarisant solide. - Soit $n$ la valence de chacun des groupes ioniques en lesquels se scinde la molécule $(n=1$ pour $\mathrm{KCl}$; $n=2$ pour $\mathrm{SO}^{\prime} \mathrm{K}^{2}$ ou pour $\left.\mathrm{ZnCl}^{2}\right)$; soit $\mathrm{F}=96.300$ coulombs, la

(1) Dunex, Potentiel thermodynamique, p. 51 ; - Ostwald, Lehrbuch der allgemeinen Chemie, t. II, p. 723. 
quantité d'électricité liée au déplacement d'un équivalent-gramme. Le passage de la quantité dm d'électricilé à travers le liquide de la pile, dans le sens $\mathrm{AB}$, ajoute à la solution $d \mathrm{~N}=\frac{d m}{n \mathrm{~F}}$ moléculesgrammes de sel, lorsque A est le pôle négatif, ce que nous supposerons ici. Alors:

$$
\frac{\partial \mathrm{N}}{\partial m}=\frac{1}{n \mathbf{F}}
$$

En transportant cette valeur dans l'équation (̋), on trouve :

$$
\frac{\partial \mathrm{E}}{\partial \gamma}=-\frac{1}{n \mathrm{~F}} \cdot \frac{1}{\gamma} \cdot \frac{\partial \bar{\sigma}}{\partial \gamma} ;
$$

et, comme $\frac{\partial \bar{\omega}}{\partial \gamma}$ est toujours positif, on conclut de là que la force électromotrice d'une pile de cette espèce varie en sens inverse de la concentration.

$2^{\circ}$ Pile constiluée par deux métaux respectivement plongés dans des solutions de leurs sels. - Le passage de $d m$, dans le même sens que ci-dessus, ajoute $\frac{1}{n \mathrm{~F}}$ molécules à la solution anodique ( $\mathrm{SO}^{\prime} \mathrm{Zn}$ dans une pile de Daniell) et en retranche le même nombre à la solution cathodique $\left(\mathrm{SO}^{4} \mathrm{Cu}\right)$. On a donc pour la première solution:

et pour la deuxième :

$$
\left(\frac{\partial N}{\partial m}\right)_{1}=\frac{1}{n \mathbf{F}}
$$

$$
\left(\frac{\partial N}{\partial m}\right)_{2}=-\frac{1}{n F}
$$

$\frac{\partial \mathrm{E}}{\partial \gamma_{1}}$ est donc négatif, et $\frac{\partial \mathrm{E}}{\partial \gamma_{2}}$ est positif; la force électromotrice d'une pile de Daniell varie dans le même sens que la concentration de la solution de $\mathrm{SO}^{4} \mathrm{Cu}$, et en sens inverse de celle de $\mathrm{SO}^{4} \mathrm{Zn}$.

$3^{\circ}$ Chaine de concentraticn de première especce. - Deux électrodes formées d'un même métal M plongent dans deux solutions inégalement concentrées de l'un de leurs sels; ces solutions seront désignées par $L_{1}$ et $L_{2}$, et numérotées dans le sens suivant lequel on les rencontre en allant du pôle A au pôle B; elles sont en contact l'une avec l'a utre, de sorte que le schéma de cette chaîne est :

$$
\mathrm{A}|\mathrm{M}| \mathrm{L}_{1}\left|\mathrm{~L}_{2}\right| \mathrm{M} \mid \mathrm{B} \text {. }
$$


I. Hittorf a montré ru'une solution électrolytique, où plongent des rlectroctes inattaquables, s'appaurrit inégalement autour de chacune d'elles. Pour 1 molécule détruite par le courant, la fraction $h$ disparait autour de la cathode, et la fraction $(1-h)$ autour de l'anode. Les fractions $h$ et $(1-h)$ s'appellent coefficients cle transport. Lorsque les électrodes sont attaquables, le chaingement définitif de concentration est la somme algébrique de l'effet produit par le phénomène de Ilittorf et de l'effet produit par la réaction entre les électrodes et les ions.

D'après cela, quand la quantité d'électricité $d m$ traverse la chaîne de $\mathrm{A}$ vers $\mathrm{B}, \frac{d m}{n \mathrm{~F}}$ molécules de sel sont détruites. La solution $\mathrm{L}_{\mathbf{1}}$ en perd, de ce chef, $(\mathbf{1}-h) \frac{d m}{n \mathrm{~F}}$, mais en gagne $\frac{d m}{n \mathbf{F}}$, qui se forment aux dépens de l'électrode, En définitive, $\mathrm{L}_{1}$ s'enrichit de

$$
\frac{d m}{n \mathbf{F}}-(1-h) \frac{d m}{n \mathbf{F}}=h \frac{d m}{n \mathbf{F}}
$$

molécules; et, par conséquent :

$$
\left(\frac{\partial \mathrm{N}}{\partial m}\right)_{1}=+\frac{h}{n \mathrm{~F}}
$$

La solution $\mathrm{L}_{2}$ perd, en même temps, $h \frac{d m}{n \mathrm{~F}}$ molécules; par conséquent:

$$
\left(\frac{\partial \mathrm{N}}{\mathrm{Im}}\right)_{2}=-\frac{h}{n \mathrm{~F}}
$$

En transportant ces valeurs dans l'équation (7), on trouve :

$$
\mathrm{E}=\frac{h}{n \mathrm{~F}}\left[-\int_{\ddot{\gamma}_{0}} \frac{1}{\gamma} \frac{\partial \sigma}{\partial \gamma} d \gamma+\int_{\tilde{\gamma}_{0}}^{\ddot{1}_{1}} \frac{1}{\gamma} \cdot \frac{\partial \sigma}{\partial \gamma} d \gamma\right],
$$

$\gamma_{0}$ désignant une concentration arbitraire, $\gamma_{1}$ et $\gamma_{2}$ les concentrations actuelles des deux dissolutions; il n'y a pas de constante à ajouter, car on doit évidemment avoir $E=$ o pour $\gamma_{1}=\gamma_{2}$. La formule cidessus se simplifie immédiatement et devient :

$$
\mathrm{E}=\frac{h}{n \mathbf{F}} \int \frac{\hat{1}^{2}}{\gamma} \cdot \frac{\partial \sigma}{\partial \gamma} \cdot d \gamma .
$$


A l'exemple de M. Nernst, nous ne pousserons le calcul plus loin qu'en supposant la dissociation complète. Alors, comme nous l'avons vu plus haut (formule 9) :

$$
\int_{\gamma_{1}}^{\gamma_{2}} \frac{1}{\gamma} \cdot \frac{\partial \pi}{d \gamma} d \gamma=j \mathrm{RT} \log \frac{\gamma_{2}}{\gamma_{1}},
$$

et définitivement :

$$
\mathrm{E}=\frac{j \mathrm{RT} h}{n \mathrm{~F}} \log \frac{\gamma_{2}}{\gamma_{1}}
$$

$4^{\circ}$ Chaine de conc nntration de deuxième espèce. - J'appelle ainsi une chaîne conforme au schéma suivant:

$$
\mathrm{A}\left|\mathrm{D} ; \mathrm{L}_{1}\right| \mathrm{L}_{2}|\mathrm{D}| \mathrm{B} \text {, }
$$

où je désigne par $\mathrm{D}$ deux électrodes constituées de la même manière par un conducteur entouré d'un dépolarisant solide, capable de fournir aux cations ce qu'il leur faut pour reconstituer des molécules (par exemple, le sel dissous étant un chlorure, le dépolarisant sera un chlorure insoluble). Des raisonnements calqués sur ceux du cas précédent conduisent à la formule :

$$
\mathrm{E}=\frac{j \mathrm{RT}(1-h)}{n \mathrm{~F}} \log \frac{\gamma_{1}}{\gamma_{2}}
$$

(A suivre.) 